\title{
PARAMETER IMPACT ON SHARING STUDIES BETWEEN UAS CNPC SATELLITE TRANSMITTERS AND TERRESTRIAL SYSTEMS
}

\author{
Robert J Kerczewski and Jeffrey D. Wilson, NASA Glenn Research Center, Cleveland, Ohio \\ William D. Bishop, Jacobs Engineering, Cleveland, Ohio
}

\begin{abstract}
In order to provide a control and non-payload communication (CNPC) link for civil-use unmanned aircraft systems (UAS) when operating in beyondline-of-sight (BLOS) conditions, satellite communication links are generally required. The International Civil Aviation Organization (ICAO) has determined that the CNPC link must operate over protected aviation safety spectrum allocations. Although a suitable allocation exists in the 5030$5091 \mathrm{MHz}$ band, no satellites provide operations in this band and none are currently planned. In order to avoid a very lengthy delay in the deployment of UAS in BLOS conditions, it has been proposed to use existing satellites operating in the Fixed Satellite Service (FSS), of which many operate in several spectrum bands. Regulatory actions by the International Telecommunications Union (ITU) are needed to enable such a use on an international basis, and indeed Agenda Item (AI) 1.5 for the 2015 World Radiocommunication Conference (WRC) was established to decide on the enactment of possible regulatory provisions.
\end{abstract}

As part of the preparation for AI 1.5, studies on the sharing FSS bands between existing services and CNPC for UAS are being contributed by NASA and others. These studies evaluate the potential impact of satellite CNPC transmitters operating from UAS on other in-band services, and on the potential impact of other in-band services on satellite CNPC receivers operating on UAS platforms. Such studies are made more complex by the inclusion of what are essentially moving FSS earth stations, compared to typical sharing studies between fixed elements. Hence, the process of determining the appropriate technical parameters for the studies meets with difficulty. In order to enable a sharing study to be completed in a less-than-infinite amount of time, the number of parameters exercised must be greatly limited. Therefore, understanding the impact of various parameter choices is accomplished through selectivity analyses. In the case of sharing studies for
AI 1.5, identification of worst-case parameters allows the studies to be focused on worst-case scenarios with assurance that other parameter combinations will yield comparatively better results and therefore do not need to be fully analyzed. In this paper, the results of such sensitivity analyses are presented for the case of sharing between UAS CNPC satellite transmitters and terrestrial receivers using the Fixed Service (FS) operating in the same bands, and the implications of these analyses on sharing study results.

\section{Introduction}

Many potential applications for civil use of UAS have been identified, with additional use concepts emerging almost daily. However, the ability of UAS to operate in the National Airspace (NAS), in particular in non-segregated airspace, faces many obstacles. The increasing pressure to remove these obstacles has resulted in the establishment of a national goal in the United States of enabling UAS to have routine access to the NAS. Among a number of technical barriers that must be overcome to meet this goal is the absence of standard, certifiable communications links supplying the CNPC function, essentially providing the link over which a pilot on the ground can control the unmanned aircraft (UA). ICAO has determined that the CNPC link must operate over a protected aviation safety spectrum allocation. Therefore such an allocation for this function, through the processes of the ITU Radiocommunication Sector (ITU-R), is being sought.

Spectrum requirements have been established for line-of-sight (LOS) and BLOS CNPC [1]. Actions taken at the 2012 WRC have established spectrum resources to address the LOS spectrum requirement. The BLOS spectrum requirement remains unfulfilled. An allocation in the 5030-5091 $\mathrm{MHz}$ band meets a portion of the requirement, however, communications satellites required to provide service in this band do not exist and none are currently planned. The UAS community has

U.S. Government work not protected by U.S. copyright 
therefore been searching for a solution to meet the BLOS CNPC needs.

It has been proposed to use existing Fixed Service Satellites (FSS), of which many operate in several bands, to provide BLOS CNPC at least until such time as dedicated satellite services in 5030-5091 $\mathrm{MHz}$ become available. Given the size of most unmanned aircraft, higher frequency bands are required in order to have antennas small enough to mount on the aircraft. Therefore the use of FSS in $\mathrm{Ku}-\mathrm{Band}(12-18 \mathrm{GHz})$ and $\mathrm{Ka}-\mathrm{Band}(26.5-40.0 \mathrm{GHz})$ for BLOS CNPC has been proposed. Military UAS operations successfully using satellite links for CNPC in these bands have been cited as demonstrating the feasibility of this approach. AI 1.5 for the 2015 WRC was established to study this proposal and the associated regulatory requirements necessary to allow such an application in those bands.

Studies on the sharing of the $\mathrm{Ku}$ - and $\mathrm{Ka}-\mathrm{Band}$ between existing services and CNPC for UAS are ongoing specifically addressing WRC-15 Agenda Item 1.5. These studies evaluate the potential impact of satellite CNPC earth station transmitters operating from UAS on other in-band services, and on the potential impact of other in-band services on satellite CNPC receivers operating on UAS platforms. In this paper we focus on the sharing studies between the UAS earth station transmitters and terrestrial services receivers, in particular the Fixed Service (FS) consisting of microwave terrestrial communications links. This portion of the studies involves the uplink (earth to satellite) portions of the $\mathrm{Ku}$ and $\mathrm{Ka}$ bands that also have an FS spectrum allocation: 14.0-14.5 $\mathrm{GHz}$ and $27.5-29.5 \mathrm{GHz}$.

As in most sharing studies, the process of determining appropriate study parameters meets with difficulty. For example: identifying comprehensive technical representations of existing incumbent systems; postulating technical characteristics of proposed new systems; selecting from sanctioned ranges of transmit and receive power levels; applying a reasonable interpretation of protection criteria; finding acceptable representations of distributions of systems; selecting the most applicable methods of aggregating system effects; selecting among a vast range of physical parameters, geographic locations, and possible system interactions; applying appropriate atmospheric and rain effects; and many other parameter selections lead to a seemingly infinite number of parameter variations. In order to enable a sharing study to be completed in a less-thaninfinite amount of time, the number of parameters exercised must be greatly limited. For the sharing study to be accepted as useful in addressing spectrum allocation requirements, the choices made for these parameter selections must be explained and defended within the ITU-R process.

Important aspects of the sharing studies such as: description of AI 1.5; UAS spectrum requirements; UAS operational scenarios applied to the sharing studies; existing incumbent in-band systems; UA earth station and incumbent system technical parameters; UA distributions; and interference protection criteria are discussed in [2]. In this paper we extend this discussion to consider the impact of several key study parameters through sensitivity analyses to validate worst-case study scenarios used to assess the compatibility of UAS earth station operations with in-band incumbent services. In addition we consider the impact of the sensitivity analysis results on sharing study outcomes.

\section{Sharing Study Parameters}

Many of the technical parameters used for the UAS-FS sharing studies for AI 1.5 are determined in other contributions to the AI 1.5 process and various ITU-R documents. For example, link budgets for the BLOS CNPC satellite communication links using the FSS have been developed for three different UA earth station antenna sizes, in order to support a variety of UA platforms, as defined in document inputs to the ITU-R Working Party 5B, responsible for AI 1.5. These link budgets define antenna transmission parameters such as transmit power and EIRP. The FS technical parameters are established by the ITU-R in other ITU-R documents such as Recommendation F.758-5 [3]. Protection criteria for FS receivers serve as the basis for analyzing the amount of potential interference from a UA earth station into an FS receiver, and thus the compatibility between UAS and FS. These are found in ITU-R Recommendations F.1494-0 and F.1495-2 [4, 5]. Operational scenarios developed by ICAO provide ranges of UA operational altitudes to be considered in sharing studies. Expected peak densities of UA operating in the airspace are defined in [1].

Thus the parameters and parameter ranges for the sharing study are defined from various accepted 
sources. The specific parameter value from the accepted ranges has a significant impact on the study outcomes and so must be selected carefully and the selection shown to be valid. In the following section we review sensitivity analyses for the following key parameters: FS station antenna azimuth; relative longitudinal position of satellite and FS station; latitude of the FS station; altitude of the UA; and elevation angle of the FS station antenna. Results of many previous analyses associated with AI 1.5 have indicated these to be the most significant parameters to be evaluated when determining the overall worst case study scenarios.

The gain pattern for the UA earth station antenna is another critical parameters for the sharing study. An ITU-R defined antenna gain useful for studies involving the FSS is found in Recommendation S.580-6 [6]. However this antenna pattern is intended for FSS earth stations on the ground, and is therefore not considered to accurately represent the back lobe structure of a UA antenna, but has been used in recent sharing studies due to its status as an accepted ITU-R antenna pattern. An antenna gain pattern more typical of the type used for UAS applications is based on a peak-envelope Bessel function, however such a gain pattern is not yet part of relevant ITU-R documentation. Therefore recent sharing studies have used both the ITU-R S.580 antenna and the peak-envelope Bessel function antenna. These antenna gain patterns are also described in the following section.

\section{Study Parameter Sensitivity Analyses}

Based on many previous analyses that have been performed during the AI 1.5 study period, the following have been identified as the key FS parameters defining the worst case interference scenario: station antenna azimuth; relative longitudinal position of satellite and FS station; latitude of the FS station; altitude of the UA; and elevation angle of the FS station antenna. These parameters have been analyzed in the order presented, and the results applied to each succeeding sensitivity analysis. This is, again, based on previous experience in performing AI 1.5 sharing studies.

Visualyse Professional software [7] was used to model the required interference scenarios and obtain resulting interference-to-noise ratios $(\mathrm{I} / \mathrm{N})$ at the $\mathrm{FS}$ receiver input. $\mathrm{I} / \mathrm{N}$ is the parameter used to characterize the required FS protection criteria.

\section{UA Earth Station Antenna Gain Pattern}

Based on link budgets developed for the CNPC BLOS link that provide the required link performance, required transmit EIRP levels have been derived for the UA earth station. From the EIRP, antenna parameters and transmit powers have been specified for antenna diameters of $0.45,0.8$ and $1.25 \mathrm{~m}$ (designated small, medium, and large) for both frequency bands under study. This range of sizes allows for various sizes of UA to be fitted with an appropriately sized antenna.

As described in the preceding section, two antenna gain patterns are being applied - the ITU-R S.580 and the peak-envelope Bessel function antenna. Figure 1 provides a comparison of these two gain patterns at $14.4 \mathrm{GHz}$ for the small and large antenna sizes. Figure 2 provides a comparison of these two gain patterns at $28.5 \mathrm{GHz}$ for the small and large antenna sizes. For the purposes of the sensitivity analyses the large version of the ITU-R S.580 was used, since either antenna gain pattern will lead to the same results. However, the next section will present sample results of sharing studies under worst case conditions for both gain patterns and antenna sizes, and difference in results reflect the differences in the gain patterns observed in Figures 1 and 2. 


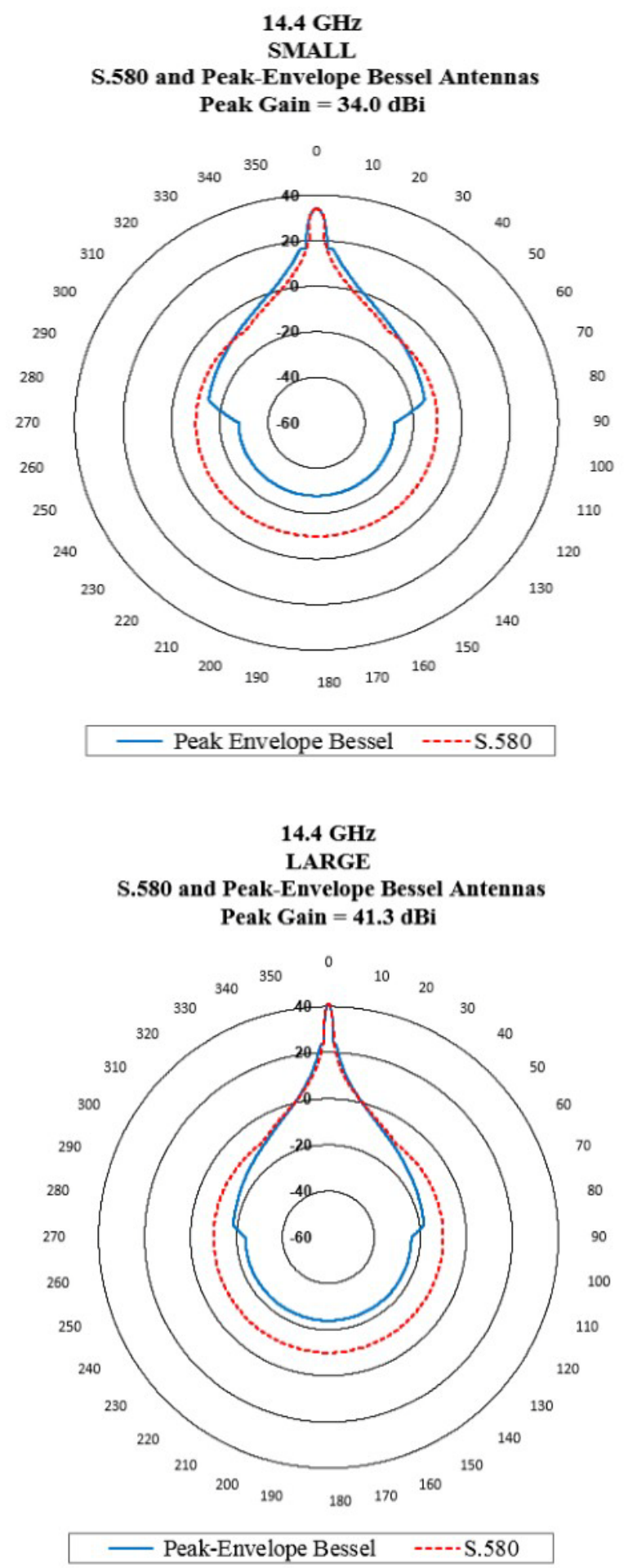

(b)

Figure 1. Antenna Patterns for the Peak-Envelope Bessel Function and S.580 Antennas at 14.4 Ghz: (a) Small Antenna; (b) Large Antenna

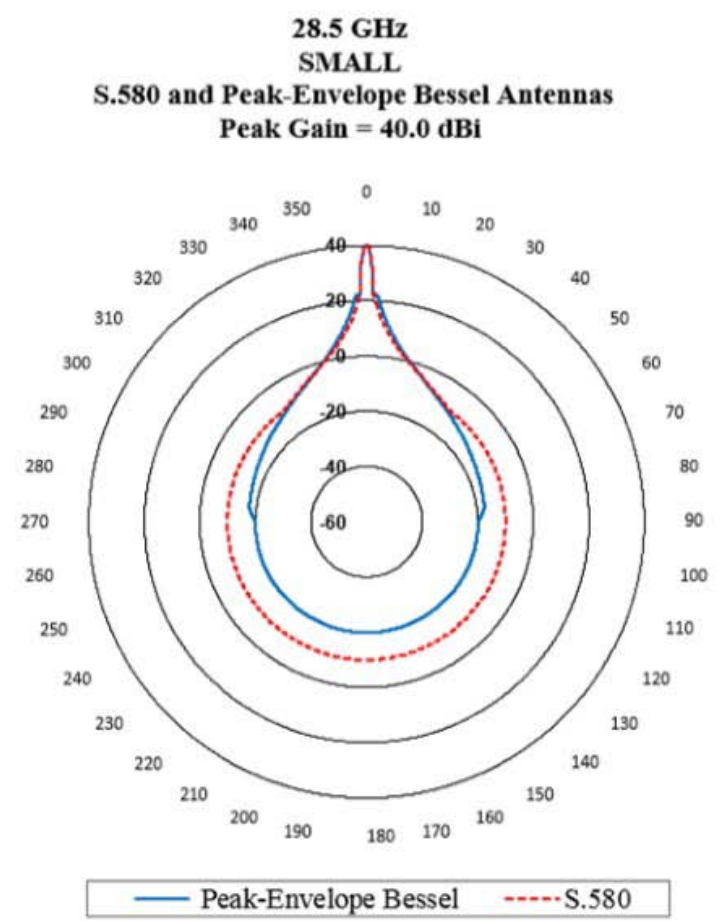

(a)

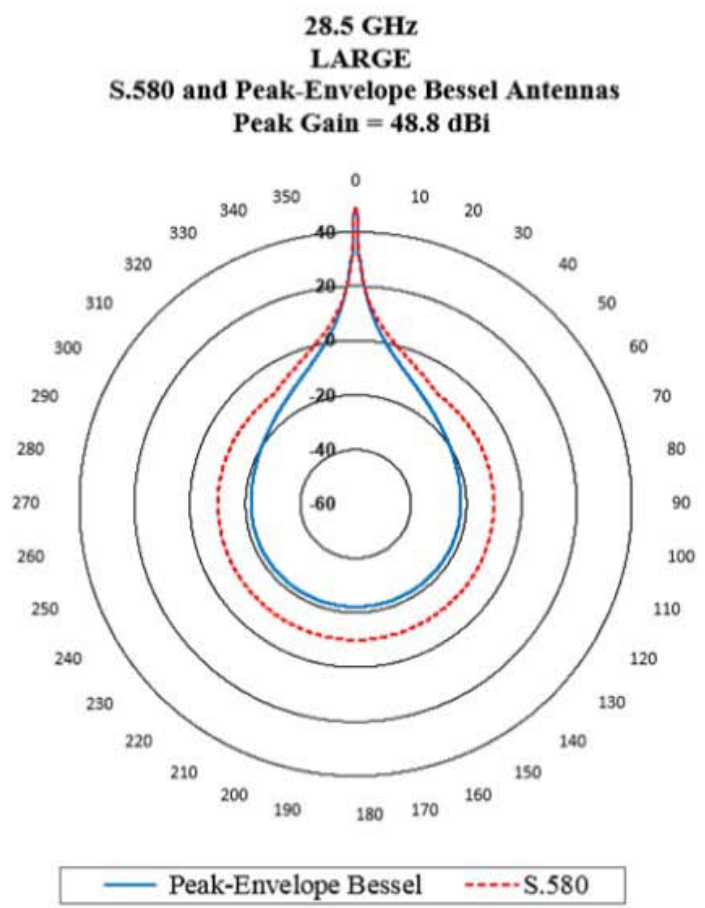

(b)

Figure 2. Antenna Patterns for the Peak-Envelope Bessel Function and S.580 Antennas at 28.5 Ghz: (a) Small Antenna; (a) Large Antenna 


\section{FS Station Antenna Azimuth and Relative Longitudinal Position of Satellite and FS Station}

FS stations consist of point-to-point and pointto-multipoint microwave terrestrial communications links transmitting digitally encoded data supporting many types of applications. The links consist of a transmitter and one or more receivers separated by a distance within radio line of sight, and can be unidirectional or bidirectional. FS stations are not limited in location or density except requiring sufficient separation in distance and/or frequency to avoid interfering with each other. The pairs of FS stations (transmitting station and receiving station) can be aligned in any direction. As a result, the FS antenna may be pointed with an azimuth range of $0^{0}$ to $360^{\circ}$, where $0^{0}$ is due North.

In the Northern Hemisphere, UA communicating with geostationary satellites will have antennas pointing generally south, offset by some amount depending on the relative location of the satellite in the geostationary arc. In the Southern Hemisphere the case is exactly opposite. It is expected that the maximum interference from the UA would be received by the FS receiver when the FS antenna azimuth is $0^{0}$.

The sensitivity analysis was performed by calculating the maximum I/N obtained with the UA at an altitude of $3000 \mathrm{ft}$. at all possible locations within the radio line of sight of the FS receiver. The FS was positioned at $70^{\circ} \mathrm{N}$ latitude and $90^{\circ} \mathrm{W}$ longitude, and the azimuth of the FS antenna was varied between $-60^{\circ}$ and $+60^{\circ}$. This was repeated for satellite positions of $90^{\circ}, 95^{\circ}, 100^{\circ}$ and $105^{\circ} \mathrm{W}$. The sensitivity analysis showed that the maximum interference does in fact occur with an FS azimuth of $0^{0}$ when the satellite at which the UA antenna is pointing is at the same longitude as the FS receiver. However, when the UA is pointing at a satellite in another location, the maximum interference is received at an FS azimuth angle offset by the difference in degrees between FS longitude and the satellite longitude. This result is depicted in Figure 3, and shows that the azimuth at which the FS antenna aligns with the pointing direction of the UA antenna produces the maximum interference into the FS receiver.

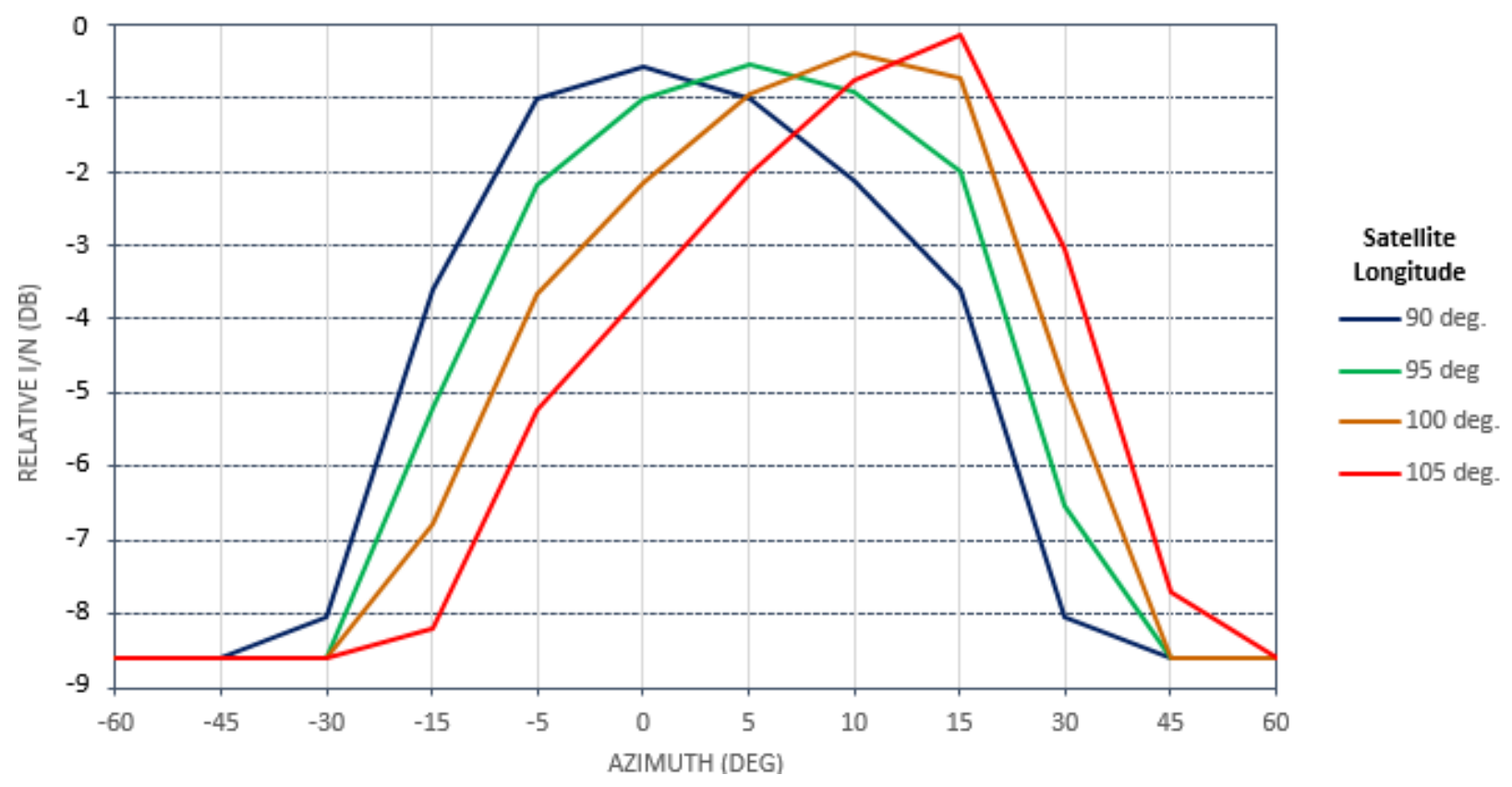

Figure 3. Relative Received I/N as a Function of FS Antenna Azimuth for Several Satellite Locations

Figure 3 also shows that the $\mathrm{I} / \mathrm{N}$ increases slightly as the satellite longitude moves farther away from the FS longitude. This is due to the elevation angle of the UA antenna being slightly lower for satellite locations farther away in longitude. An operational limitation of the UA CNPC satellite link 
is imposed by the elevation angle of the UA antenna; below angles of about $10^{\circ}$ shadowing from the body of the aircraft renders the satellite link very difficult to maintain. Therefore, when using satellites located at longitudes different from the UA location, a minimum elevation angle must be maintained, requiring operation at a lower latitude. This negates the slight difference in $\mathrm{I} / \mathrm{N}$ as observed in Figure 3 and we can state that the worst case scenario for UA interference into the FS receiver can be effectively modeled with the FS station and satellite at the same longitude and the FS antenna azimuth at $0^{\circ}$.

\section{Latitude of the FS Station}

An FS station can be interfered with by UA that are located within its radio line of sight, which is a function of both the distance and altitude of the UA relative to the FS. Beyond the line of sight, interference from the UA into the FS receiver is negligible. Therefore, the location in latitude of the
FS will determine the location in latitude of UA that can create interference.

As the latitude of UA increases, the elevation angle of the UA antenna decreases, with the result that UA antenna sidelobe levels directed toward the ground are higher. For this reason it is expected that the interference from the UA into the FS receiver should increase as the latitude increases.

The sensitivity analysis for FS station latitude was performed with the satellite and FS at the same longitude and an FS antenna azimuth of $0^{\circ}$. The maximum $\mathrm{I} / \mathrm{N}$ obtained with the UA at an altitude of $3000 \mathrm{ft}$. at all possible locations within the radio line of sight of the FS receiver was calculated at latitudes of $10^{\circ}, 20^{\circ}, 30^{\circ}, 40^{\circ}, 50^{\circ}, 60^{\circ}$, and $70^{\circ}$.

The results of the analysis are shown in Figure 4. As expected, the interference increases with increasing latitude. The worst case latitude is established by these results as $70^{\circ}$.

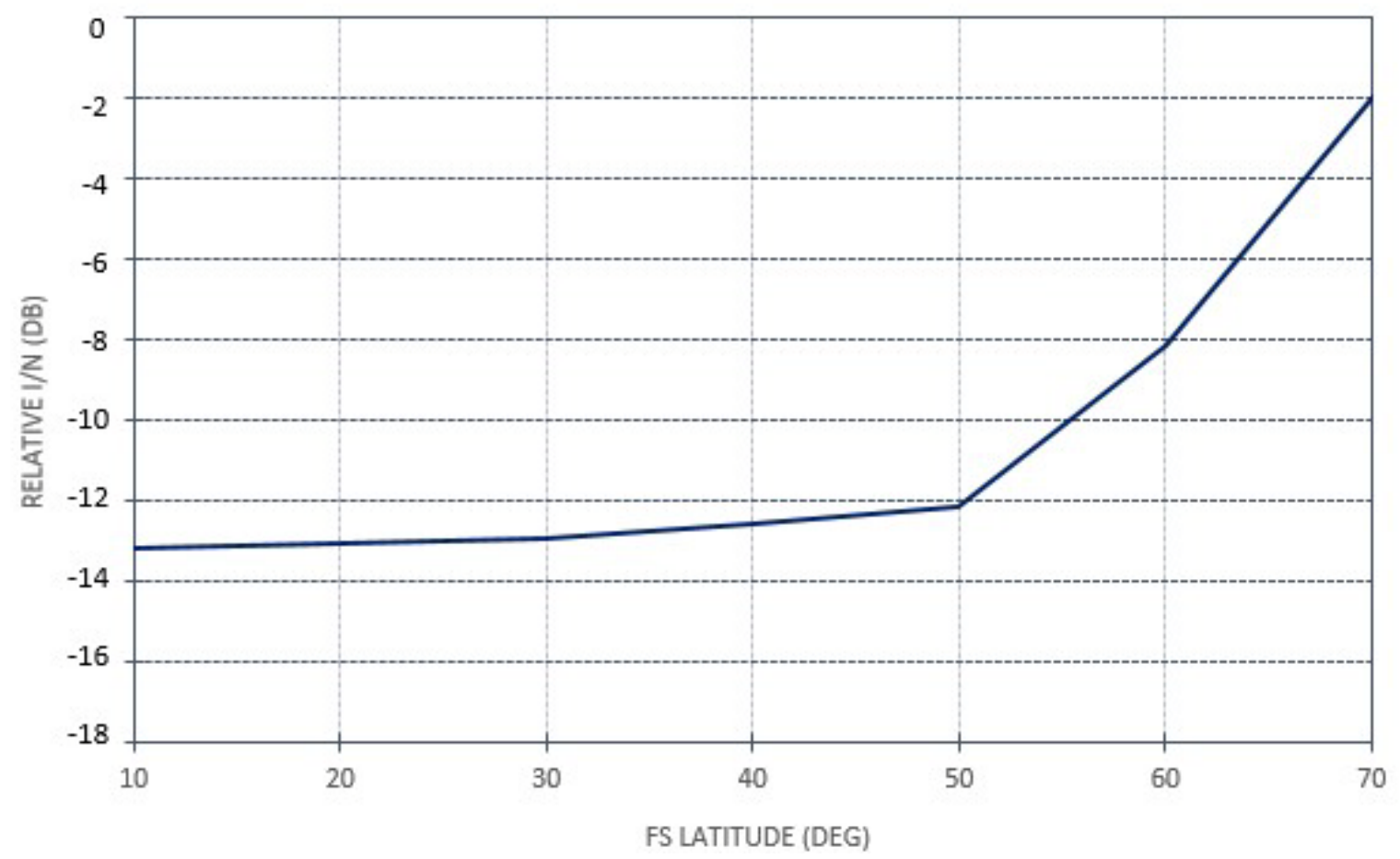

Figure 4. Relative Received I/N as a Function of FS Latitude

\section{Altitude of the $U A$}

In the UA operational scenarios defined by ICAO that are relevant to the UAS-FSS sharing studies, the altitude can range from 500 to $66000 \mathrm{ft}$.
However, altitudes below $3000 \mathrm{ft}$. have not been considered in these sharing studies Operations with UA using satellite communications for BLOS CNPC would not use these systems for takeoff, landing, departure and arrival operations due to the latency of 
the satellite links exceeding the requirements for such operations. It was not necessary to study altitudes above $19000 \mathrm{ft}$. because satellite communications would be unusable for all but a few UA if operations were restricted to altitudes above $19000 \mathrm{ft}$.

The sensitivity analysis for UA altitude was performed with the satellite and FS at the same longitude. Two latitudes, $40^{\circ}$ and $70^{\circ}$, were tested with an FS antenna azimuth of $0^{\circ}$. The maximum $\mathrm{I} / \mathrm{N}$ obtained with the UA at all possible locations within the radio line of sight of the FS receiver was calculated at altitudes of 3000 to $19000 \mathrm{ft}$. in $2000 \mathrm{ft}$. increments.
At higher altitudes, the UA must be farther from the FS receiver in order to have the relative angle into the FS antenna as at lower altitudes. Hence, at higher altitudes, the distance between the UA and FS to obtain the same level of UA antenna sidelobe coupling is increased, resulting in greater propagation loss.

The results shown in Figure 5 confirm this behavior. The highest interference occurs at the lowest altitude. Therefore, for the sharing studies, a UA altitude of $3000 \mathrm{ft}$. correspond to the worst case.

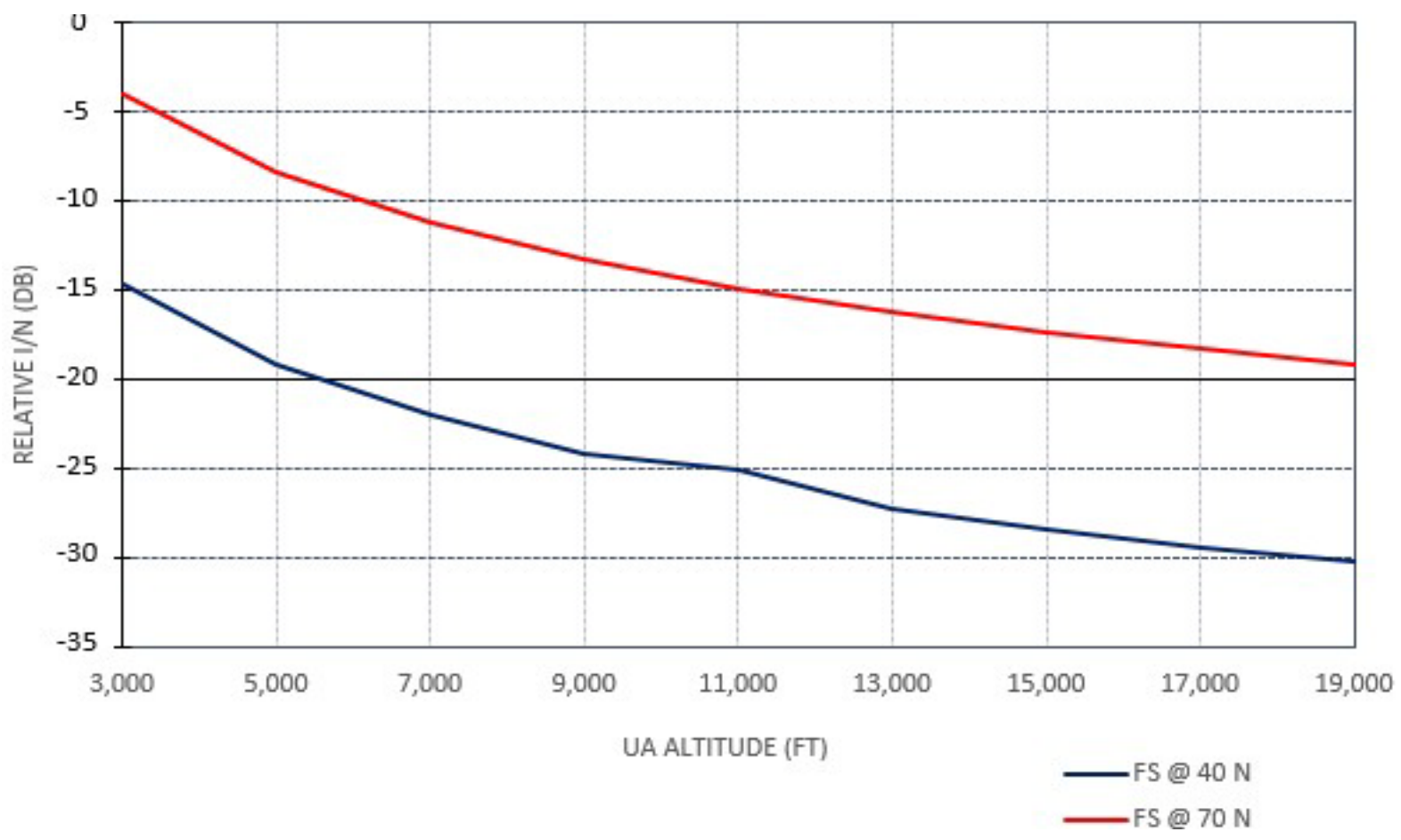

Figure 5. Relative Received I/N as a Function of UA Altitude at Two FS Latitudes

\section{Elevation Angle of the FS Station Antenna}

The final parameter to be analyzed is the FS antenna elevation angle. Although there can be exceptional cases of higher or lower elevation angle for an FS antenna, the range over which FS antenna elevation angle must be considered for sharing studies is $\pm 5^{0}$.

For this sensitivity analysis, the satellite and FS at the same longitude. Two latitudes, $40^{\circ}$ and $70^{\circ}$, were tested with the UA at an altitude of $3000 \mathrm{ft}$. The maximum I/N obtained with the UA at all possible locations within the radio line of sight of the FS receiver was calculated for $\mathrm{FS}$ antenna elevation angles from $-5^{0}$ to $+5^{0}$, in $1^{0}$ increments

For a fixed UA location, it is expected that the interference received by the FS would increase as the FS antenna elevation angle is increased, since the main lobe of the FS antenna would be coupling into a higher UA antenna sidelobe level.

The results plotted in Figure 6 show that the highest interference levels occur at the maximum FS antenna elevation angle. Therefore, the worst case FS antenna elevation angle is considered to be $+5^{0}$. 


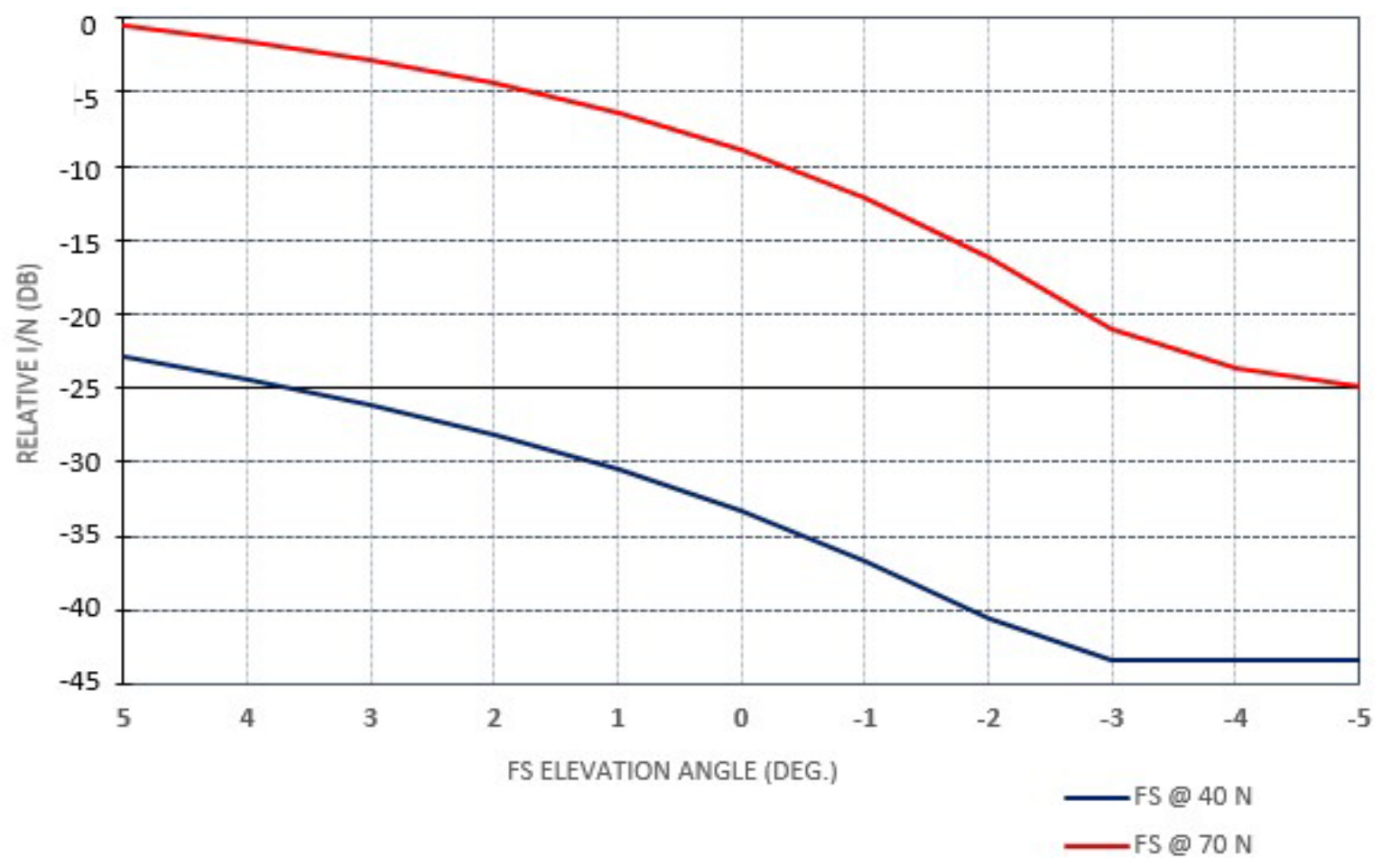

Figure 6. Relative Received I/N as a Function of FS Antenna Elevation Angle at Two FS Latitudes

\section{Worst Case Study Parameters}

To summarize the results of the sensitivity analyses, the worst case interference scenario is defined by the following parameters:

- FS antenna azimuth $=0^{0}$ for the case where the satellite and FS station are located at the same longitude

- FS latitude $=70^{\circ}$

- UA altitude $=3000 \mathrm{ft}$.

- FS antenna elevation angle $=+5^{0}$

\section{Sharing Study Results - Examples}

The worst case scenario defined by the parameters determined by the preceding sensitivity analyses was applied two create two sharing study examples, one for each frequency band under study.

The long term FS protection criteria provides the maximum allowable interference into the FS receiver, expressed as I/N. From ITU-R F.758-5 [3], the I/N must not exceed $-10 \mathrm{~dB}$ for more than $20 \%$ of the time. This protection criteria applies to both 14.014.5 $\mathrm{GHz}$ and 27.5-29.5 GHz. It considers the aggregate interference from all interferers.

Figure 7 shows the geometric layout of the interference scenario. A UA located at the center of the concentric circles is maintaining a CNPC link through a satellite. The antenna gain pattern of the UA antenna is shown in the blue trace. FS locations (shown as radio towers along the bottom of the figure) show how the angular relationship between the FS station and the UA earth station antenna varies. The elevation angle of the UA antenna is noted as well as the angle below the UA horizon, equivalent to the arrival angle of the interfering signal at the FS antenna. The intersection point of the line from the FS to the UA with the UA antenna gain pattern varies with the relative location, resulting in different UA antenna sidelobe levels directed toward the FS. 


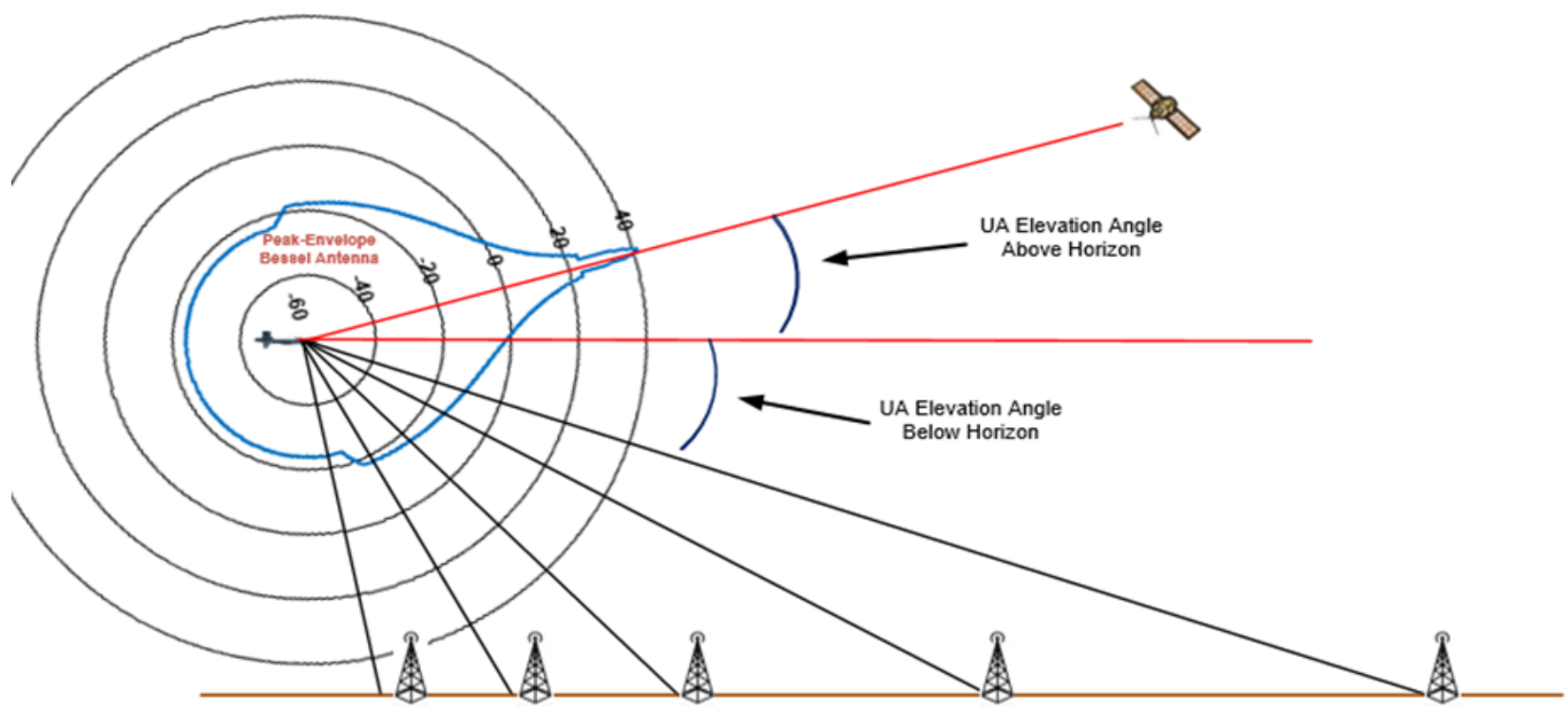

Figure 7. UA - FS Interference Scenario

The example long-term interference analysis results are shown in Figure 8 (for the 14.0-14.5 GHz) and Figure 9 (for the 27.5-29.5 GHz case). Each of these figures shows the cumulative distribution function (cdf) for $\mathrm{I} / \mathrm{N}$ resulting from UA locations within the line of sight of the FS receiver. Each figure contains four curves, one each for the small and large version of the ITU-R S.580 antenna pattern and one each for the small and large version of the peak-envelope Bessel antenna. The previously determined worst case conditions were applied. The expected peak density of UA is taken into account, as well as the probability of a UA and FS operating at the same frequency, in calculated the cdfs.

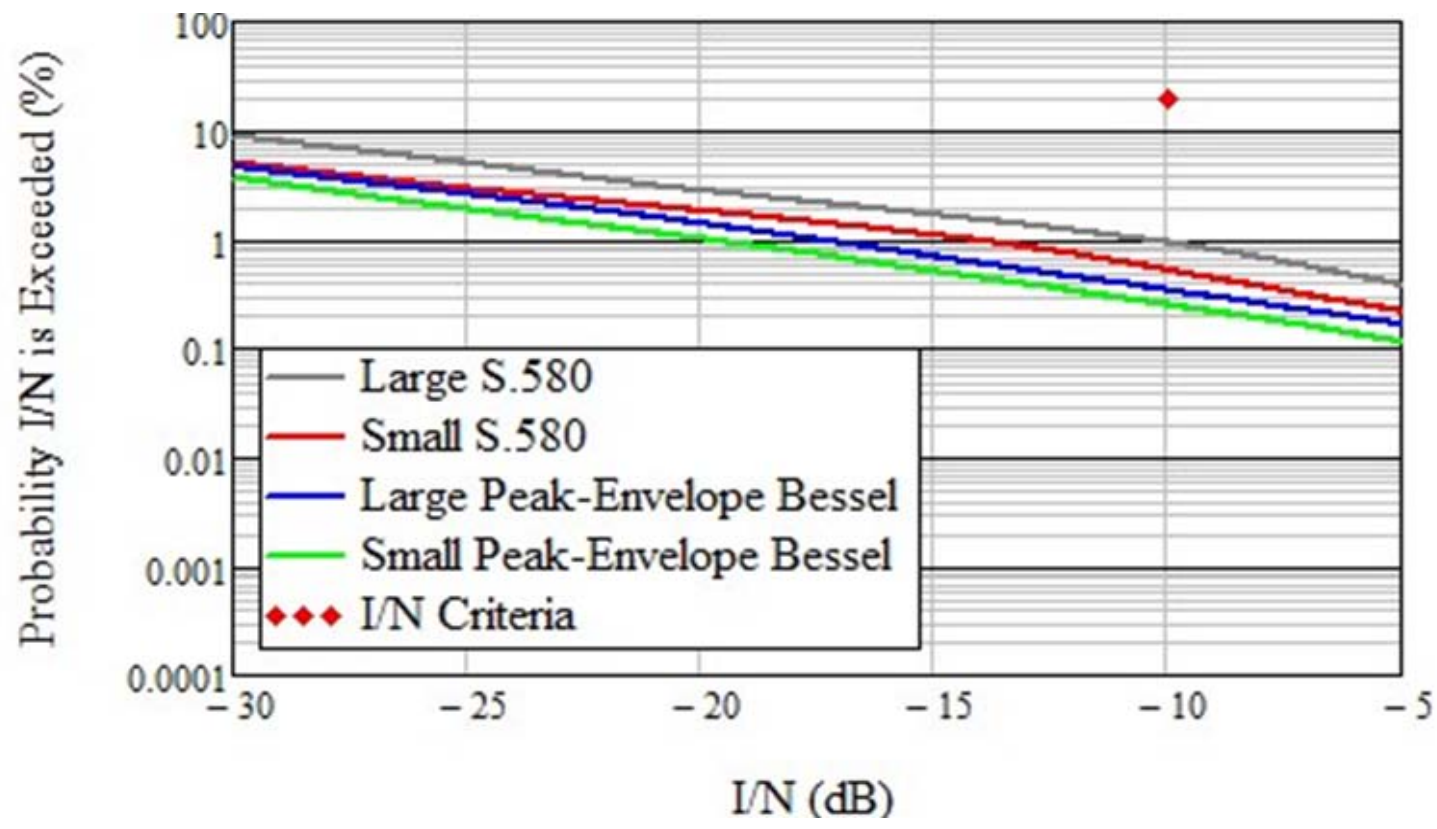

Figure 8. Worst Case cdfs for 14.0-14.5 Ghz 


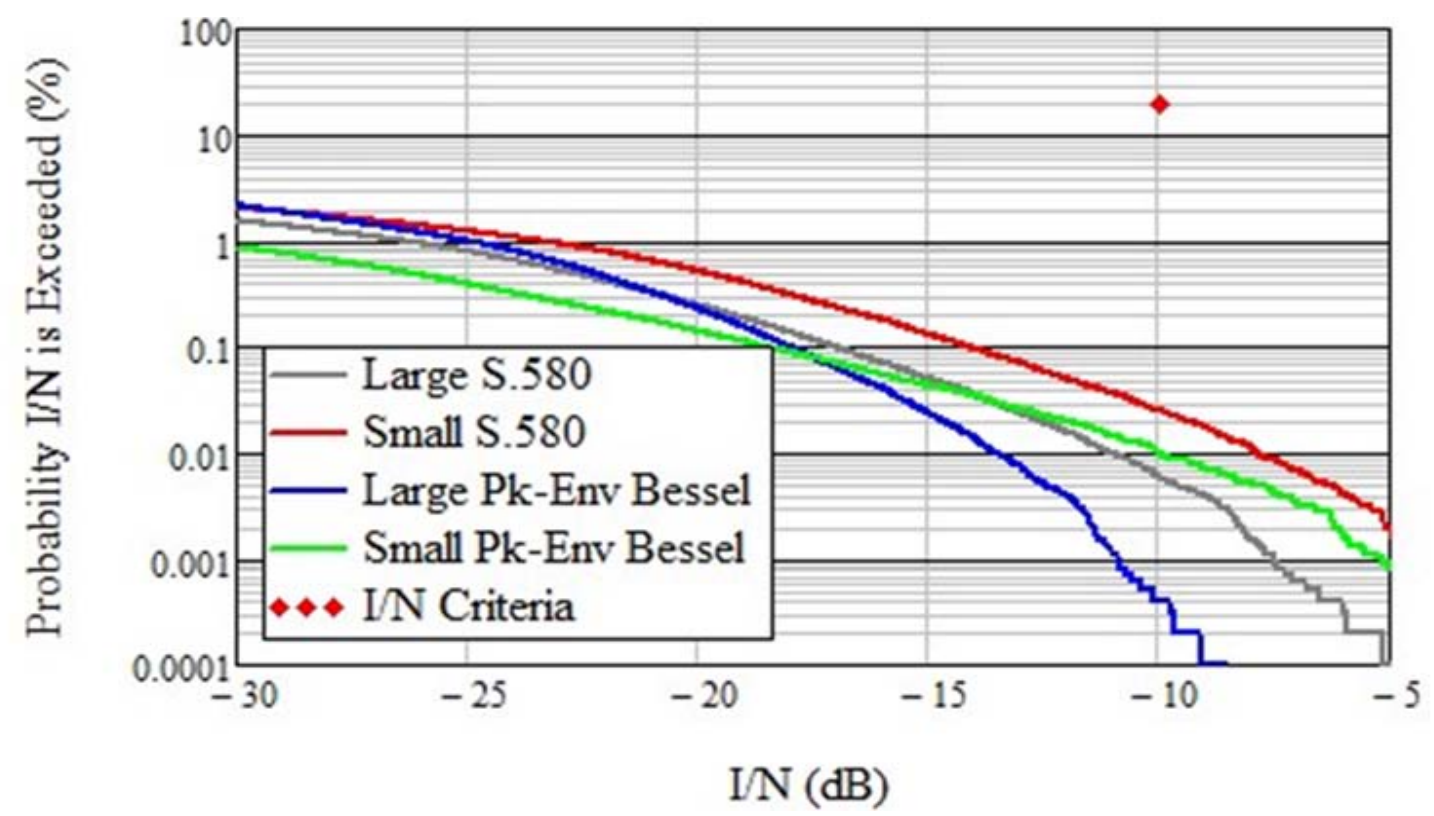

Figure 9. Worst Case cdfs for 27.5-29.5 Ghz

The protection criteria is noted by a red diamond in each figure. For both frequency bands, the results show that the long term protection criteria is not violated. Since these results are for the worst case scenario, interference levels will be lower for all other cases (e.g. lower latitudes, higher altitudes, lower FS antenna elevation).

The 14.0-14.5 GHz I/N curves are considerably higher than the $27.5-28.5 \mathrm{GHz}$ curves, due to the narrower main lobe of the antenna pattern obtained for the same size antennas at the higher frequency, as well as a lower transmit power used at 27.5-29.5 $\mathrm{GHz}$, resulting in relatively lower sidelobe levels creating interference into the FS receiver. For both frequency ranges, the peak-envelope Bessel produces lower interference than the ITU-R S.580 of the same size, due to lower sidelobe levels. (See Figures 1 and 2).

For the 14.0-14.5 GHz case, the peak-envelope Bessel antenna produces lower I/N than the ITU-R S.580 antenna, and for both gain patterns the large antenna creates a higher $\mathrm{I} / \mathrm{N}$ than the small antenna. For the $27.5-29.5 \mathrm{GHz}$ case the small antennas create higher interference levels than the large antennas of the same gain pattern.

The antenna gain pattern applied in the sharing studies has a significant impact on the resulting $\mathrm{I} / \mathrm{N}$ at the FS receiver. For the sharing study involving the
UA transmitter and FS receiver which is the subject of this paper, the difference, while significant, does not change the result that the long term FS protection criteria are still met; due to the expected peak density of UA it is unlikely that more than one UA will be contributing interference to an FS receiver at a given time so the difference between antenna patterns is not aggregated over many interfering paths. For the sharing study involving FS transmitters interfering into UA receivers, the difference between the two antenna patterns is much more significant since the UA will "see" interference aggregating from many FS transmitters within its line of sight in locations where there is a high density of FS stations.

\section{Conclusions}

In order for the 2015 WRC to consider the use of the FSS for UAS BLOS CNPC links under Agenda Item 1.5, sharing studies between UA and incumbent terrestrial services, in particular the FS, are being performed. These studies will indicate whether sharing of the spectrum between UA operating over the FSS and FS is feasible. Due to the mobile nature of UA, the sharing studies are more complicated than for cases where mobile systems are not involved.

As part of the sharing studies it is necessary to consider the worst case interference situation. In this 
paper we have analyzed the key study parameters to verify the FS latitude, FS antenna azimuth and elevation angles, UA altitude and relative longitudinal position of the FS and satellite to define the worst case interference scenario. We then presented example sharing studies results addressing the long term FS protection criteria. In these examples, the protection criteria are met, although results vary depending on the UA antenna size and gain pattern applied.

Sharing studies analyzing the worst case interference scenario for the case of UA earth stations transmitters interfering into FS receivers can now be completed and contributed to the deliberations on Agenda Item 1.5 at the 2015 WRC.

\section{References}

[1] ITU-R Report M.2171 - Characteristics of unmanned aircraft systems and spectrum requirements to support safe operation in nonsegregated airspace.

[2] Kerczewski, R.J., Wilson, J. D. and Bishop, W. D., "Assessing spectrum compatibility for beyondline-of-sight UAS control and non-payload communications", 2014 ICNS Conference, April 2014.
[3] ITU-R Recommendation F.758-5, System parameters and considerations in the development of criteria for sharing or compatibility between digital fixed wireless systems in the fixed service and systems in other services and other interference, March 2012.

[4] ITU-R Recommendation F.1494-0, Interference criteria to protect the fixed service from time varying aggregate interference from other services sharing the $10.7-12.75 \mathrm{GHz}$ band on a co-primary basis, May 2000 .

[5] ITU-R Recommendation F.1495-2, Interference criteria to protect the fixed service from time varying aggregate interference from other radiocommunication services sharing the 17.7-19.3 $\mathrm{GHz}$ band on a co-primary basis, March 2012.

[6] ITU-R Recommendation S.580-6, Radiation diagrams for use as design objectives for antennas of earth stations operating with geostationary satellites, January 2004.

[7]

http://transfinite.com/content/Professional.html

2015 Integrated Communications Navigation and Surveillance (ICNS) Conference

April 21-23, 2015 